\title{
The effects of fulvestrant, an estrogen receptor antagonist, on the proliferation, differentiation and mineralization of osteoprecursor cells
}

\author{
JUN-BEOM PARK \\ Department of Periodontics, Seoul St. Mary's Hospital, College of Medicine, \\ The Catholic University of Korea, Seoul 137-701, Republic of Korea
}

Received August 30, 2012; Accepted November 19, 2012

DOI: $10.3892 / \mathrm{mmr} .2012 .1200$

\begin{abstract}
Fulvestrant is a novel type of endocrine treatment and is considered to be a potent inhibitor of breast cancer cell proliferation. Fulvestrant is reported to work by downregulating as well as degrading the estrogen receptor, leading to an inhibition of estrogen signaling through the estrogen receptor. The effects of various doses of fulvestrant for bone cells have not yet been fully investigated. In the present study, the effects of fulvestrant on osteoprecursor cells were evaluated. The effect on cell viability was determined using the 3-(4,5-dimethylthiazol-2-yl)-2,5-diphenyltetrazolium bromide (MTT) assay and protein measurement. Differentiation and mineralization were examined using an alkaline phosphatase activity (ALP) test and Alizarin red S staining. The protein expression of osteocalcin was evaluated using western blot analysis. Cultures grown in the presence of fulvestrant at concentrations of $0.1-10 \mu \mathrm{M}$ did not show any significant change in cell proliferation. Cultures grown in the presence of fulvestrant showed a dose-dependent reduction in ALP activity, however, statistically significant differences were not achieved. Cultures grown in the presence of fulvestrant presented with a dose-dependent reduction in mineralization with a statistically significant difference at the $10 \mu \mathrm{M}$ concentration. The use of fulvestrant may produce negative effects on the mineralization of osteoprecursor cells, while long-term use of fulvestrant may have detrimental effects on osteoblastic activity.
\end{abstract}

\section{Introduction}

Estrogen has been shown to exert a variety of pleiotropic effects in target tissues as diverse as bone, breast, blood vessel,

Correspondence to: Dr Jun-Beom Park, Department of Periodontics, Seoul St. Mary's Hospital, College of Medicine, The Catholic University of Korea, 222 Banpo-daero, Seocho-gu, Seoul 137-701, Republic of Korea

E-mail: jbassoonis@yahoo.co.kr

Key words: differentiation, fulvestrant, mineralization, osteoblast, proliferation brain and the male and female gonads (1). Estrogen plays an important role in the regulation of bone remodeling and maintenance of the skeleton $(2,3)$.

The ability of estrogens to stimulate the transcriptional activity of the estrogen receptor may be inhibited by a diverse range of estrogen antagonists (4). Fulvestrant, or ICI 182,780, is a $7 \alpha$-alkylsulphinyl analog of estradiol that competes with endogenous estrogen for binding to the estrogen receptor (5). Fulvestrant is a novel type of endocrine treatment and is considered to be a potent inhibitor of breast cancer cells (6). The binding of fulvestrant to the estrogen receptor appears to prevent receptor dimerization and impairs energy-dependent nucleo-cytoplasmic shuttling, consequently blocking nuclear localization of the receptor (4,7). Additionally, the fulvestrant-estrogen receptor complex that enters the nucleus is transcriptionally inactive since the two activation domains AF-1 and -2 are disabled (8). Thus, fulvestrant works by downregulating as well as degrading the estrogen receptor, leading to an inhibition of estrogen signaling through the estrogen receptor $(5,9)$.

Previous studies investigating the effects of fulvestrant on cell proliferation and differentiation have yielded inconsistent results. Low doses of fulvestrant have been shown to inhibit the proliferation of the MCF-7 breast cancer cell line (10) and to inhibit insulin-like growth factor-1 that stimulates cell proliferation (11). A concentration of $0.1 \mu \mathrm{M}$ of fulvestrant was reported to increase the proliferation of the human fetal osteoblastic cell line, expressing high levels of estrogen receptors (hFOB/ER9) (12). However, no statistically significant difference occurred in the number of cells in the treated $(0.1$ or $10 \mu \mathrm{M}$ of fulvestrant) and untreated groups using calvarial osteoblasts (9). The conflicting responses to fulvestrant may be partly attributed to the species differences, the system model, the stage of differentiation of the cells, the culturing period and variable estrogen receptor content among various cell lines and primary cultures $(12,13)$. Moreover, limited studies are available with regard to the effects of fulvestrant on the differentiation and mineralization of osteoblasts (9). The effects of various doses of fulvestrant for bone cells have not yet been fully investigated.

The present study aimed to examine the effects of the fulvestrant at varying doses $(0.01-10 \mu \mathrm{M})$ on cell proliferation, differentiation and the mineralization of preosteoblasts. Viability was evaluated using the 3-[4,5-dimethylthiazol-2-yl]- 
2,5-diphenyltetrazolium bromide (MTT) reagents, and the alkaline phosphatase activity (ALP) test and Alizarin red S staining were used to assess the differentiation and mineralization of treated cells, respectively. The expression of proteins associated with bone formation and osteocalcin was evaluated using a western blot analysis.

\section{Materials and methods}

Cell culture. MC3T3-E1 murine calvarial preosteoblasts were maintained in $\alpha$-minimum essential medium ( $\alpha$ MEM; Invitrogen, Carlsbad, CA, USA) supplemented with $10 \%$ fetal bovine serum (Invitrogen) and antibiotics $(100 \mathrm{U} / \mathrm{ml}$ of penicillin and $100 \mu \mathrm{g} / \mathrm{ml}$ of streptomycin; Invitrogen). Culture media were changed to an osteogenic differentiation medium [ $\alpha$ MEM supplemented with $50 \mu \mathrm{g} / \mathrm{ml}$ ascorbic acid (Sigma-Aldrich, St. Louis, MO, USA)] and $10 \mathrm{mM}$ $\beta$-glycerolphosphate (Sigma-Aldrich) to induce osteogenic differentiation. Cells were maintained at $37^{\circ} \mathrm{C}$ in a humidified $5 \% \mathrm{CO}_{2}$ environment. The culture media were replenished with fresh media every three to four days. Fulvestrant, or ICI 182,780, was dissolved in dimethyl sulfoxide (DMSO; Sigma-Aldrich) and filter-sterilized. An equal amount of DMSO was applied in the control and test groups to minimize the effects of DMSO on cell growth and for differentiation between the control and treated cultures. The final concentration of DMSO in the culture never exceeded $0.01 \%$.

Cell proliferation. Cells were plated at a density of $1.0 \times 10^{4} \mathrm{cells} / \mathrm{ml} /$ well in 12 -well plates and the cultures were stimulated with fulvestrant at final concentrations ranging from 0.01 to $10 \mu \mathrm{M}$. The effects of fulvestrant on the proliferation of the preosteoblasts were assessed at Day 4. Cells were incubated for $1 \mathrm{~h}$ with the MTT reagent with a final concentration of $0.5 \mathrm{mg} / \mathrm{ml}$ (13). Rinsing with phosphate-buffered saline (PBS, pH 7.4) was followed by the addition of DMSO. After complete dissolution with gentle agitation, aliquots were transferred into 96-well plates and absorbance was recorded at 560 and $670 \mathrm{~nm}$ using the microplate spectrophotometer system (BioTek, Winooski, VT, USA).

Protein measurement. Cells were plated and cultured in $\alpha \mathrm{MEM}$ in the presence of ascorbic acid and $\beta$-glycerolphosphate for 14 days. Protein content was determined using the Coomassie Plus Protein Assay Reagent (Pierce Biotechnology, Rockford, IL, USA). Aliquots of standard bovine serum albumin (BSA) or a sample were mixed with the Coomassie reagent and the absorbance was measured at $595 \mathrm{~nm}$ using the microplate spectrophotometer system. The results are presented as a percentage of the control values.

ALP activity assays. The ALP assay for osteoblast differentiation was performed at Day 14. Cells were lysed with a buffer containing $10 \mathrm{mM}$ Tris- $\mathrm{HCl} \mathrm{pH} 7.4$ and $0.2 \%$ Triton $\mathrm{X}-100$ and were then sonicated for $20 \mathrm{sec}$ at $4^{\circ} \mathrm{C}$. Samples were incubated with $10 \mathrm{mM}$ p-nitrophenylphosphate as a substrate in $100 \mathrm{mM}$ glycine buffer ( $\mathrm{pH} 10.5$ ) containing $1 \mathrm{mM} \mathrm{MgCl}_{2}$ at $37^{\circ} \mathrm{C}$ in a water bath (14). Total protein content was determined in comparison with a series of BSA as the internal standards. The absorbance at $405 \mathrm{~nm}$ was measured using a microplate reader, and ALP activities were normalized with respect to total protein content (15).

Mineralization assay. A mineralized matrix was detected using Alizarin red S staining for qualitative analysis (9). Cells were washed twice with PBS, fixed with $70 \%$ ethanol in ice-cold PBS for $1 \mathrm{~h}$ and rinsed twice with deionized water. The cultures were stained with $40 \mathrm{mM}$ Alizarin red S (Sigma-Aldrich) for 30 min under gentle agitation. For quantification, cells stained with Alizarin red $\mathrm{S}$ were destained with $10 \%$ cetylpyridinium chloride by agitation. Extracted stain (200 $\mu \mathrm{l}$ ) was transferred to a 96-well plate and the absorbance $562 \mathrm{~nm}$ was determined spectrophotometrically.

Western blot analysis. Cells were washed twice with ice-cold PBS and solubilized with lysis buffer containing $10 \mathrm{mM}$ Tris- $\mathrm{HCl} \mathrm{pH} 7.4,0.2 \%$ Triton $\mathrm{X}-100$. The lysates were centrifuged at $14,000 \mathrm{rpm}$ for $20 \mathrm{~min}$ at $4^{\circ} \mathrm{C}$ to remove the nuclear pellet. The supernatants were boiled in a sodium dodecyl sulfate sample buffer containing $\beta$-mercaptoethanol. Equal amounts of cell extracts were separated by sodium dodecyl sulfate-polyacrylamide gel electrophoresis and then transferred onto polyvinylidene fluoride microporous membrane (Immobilon-P membranes; Millipore Corporation, Billerica, MA, USA). Membranes were then blocked in $0.1 \%$ (v/v) PBS and Tween-20 containing 5\% (w/v) powdered milk for at least $1 \mathrm{~h}$. The membrane was immunoblotted with the desired mouse antibodies against osteocalcin (Santa Cruz Biotechnology, Inc., Santa Cruz, CA, USA) diluted in the same buffer at the recommended concentrations. The membrane was incubated with horseradish peroxidase-conjugated secondary antibody and then the washed blot was developed using enhanced chemiluminescence detection kits (16).

Statistical analysis. Results are presented as the mean \pm standard deviation of the experiments. One-way analysis of variance (ANOVA) was performed to determine differences between groups using commercially available software (SPSS 12 for Windows, SPSS, Inc., Chicago, IL, USA). P<0.05 was considered to indicate a statistically significant difference.

\section{Results}

Cell proliferation. Cultures grown in the presence of fulvestrant at a concentration of 0.1 to $10 \mu \mathrm{M}$ did not show any significant change in cell proliferation (Fig. 1).

Viability/proliferation of the cells with protein measurement. The protein concentration of the cultures grown in osteogenic differentiation medium at Day 14 did not show any significant differences between the observed groups (Fig. 2).

ALP activity assays. Cultures growing in the absence of fulvestrant exhibited the highest value for the ALP activity. Cultures grown in the presence of fulvestrant showed dose-dependent reductions in ALP activity, however, statistically significant differences were not achieved in the observed groups (Fig. 3).

Mineralization/calcium deposition assay. Cultures without fulvestrant showed the highest rate of mineralized nodule 


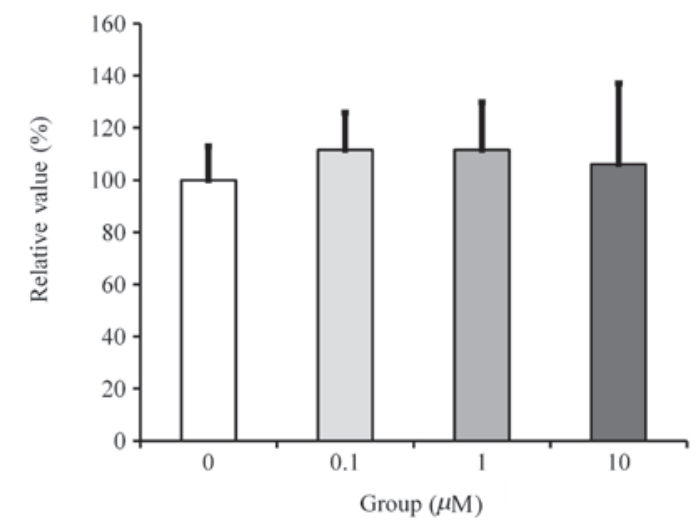

Figure 1. Determination of cell proliferation using the MTT assay at Day 4.

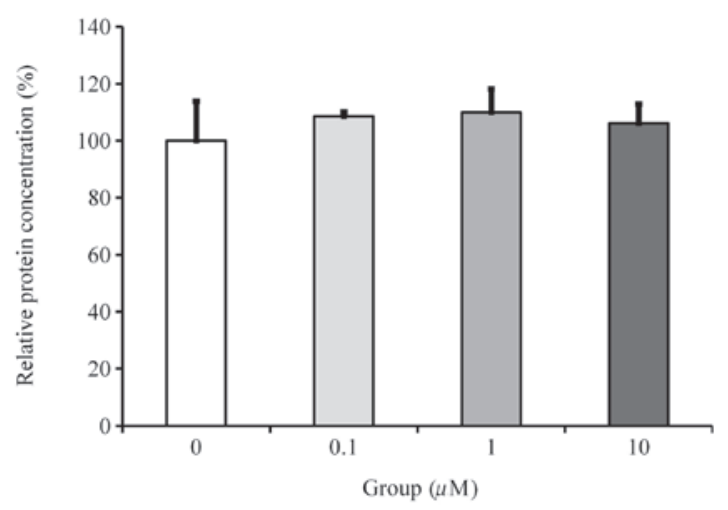

Figure 2. Viability of cells cultured in osteogenic media at Day 14 with protein measurement.

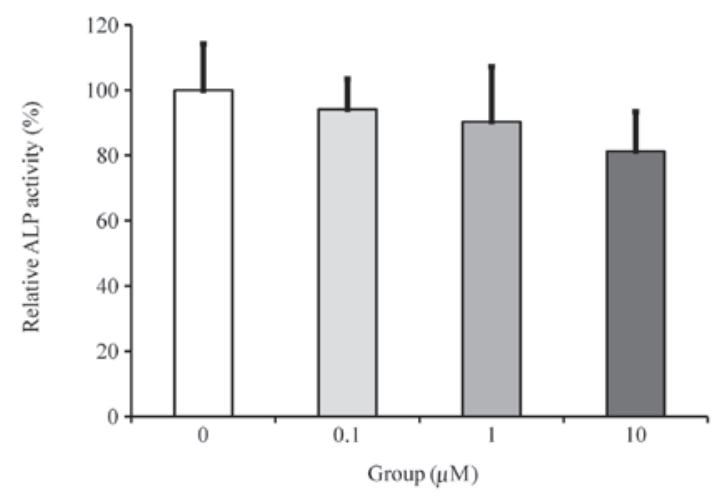

Figure 3. Relative value of alkaline phosphatase (ALP) activity.

formation. Results showed that cultures grown in the presence of fulvestrant presented with a dose-dependent reduction in mineralization. However, statistically significant differences were observed only in the $10 \mu \mathrm{M}$ concentration group (Fig. 4).

Western blot analysis. Western blot analysis was performed to detect protein expression following treatment with fulvestrant (Fig. 5). The results showed that the addition of fulvestrant decreased the expression of osteocalcin (Fig. 6). Normalization of the protein expression demonstrated that the group treated with $10 \mu \mathrm{M}$ fulvestrant yielded $83.8 \pm 6.5 \%$, when cultures without fulvestrant were considered to be

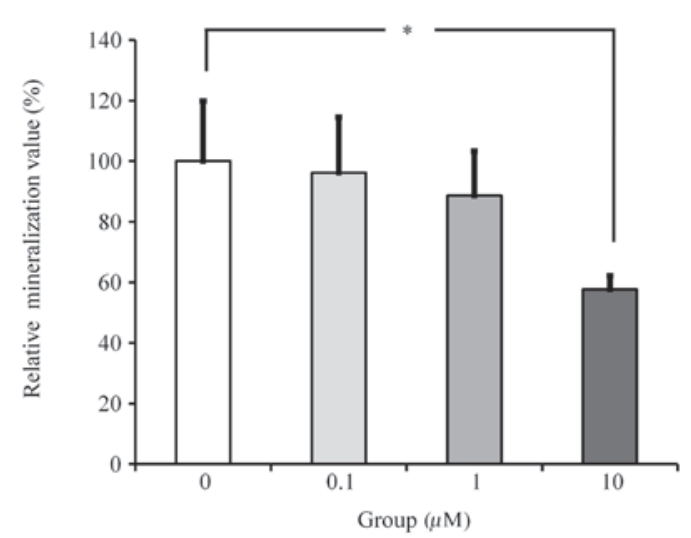

Figure 4. Relative value of mineralization following the addition of fulvestrant in the cultures. *A statistically significant difference was observed when compared with the control (non-loaded group; $\mathrm{P}<0.05$ ).

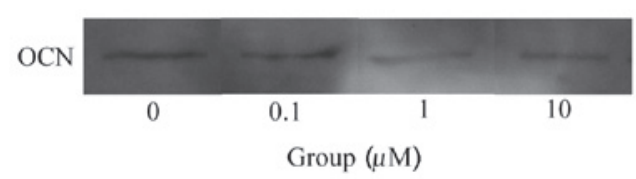

Figure 5. Western blot analysis to detect the protein expressions of osteocalcin $(\mathrm{OCN})$.

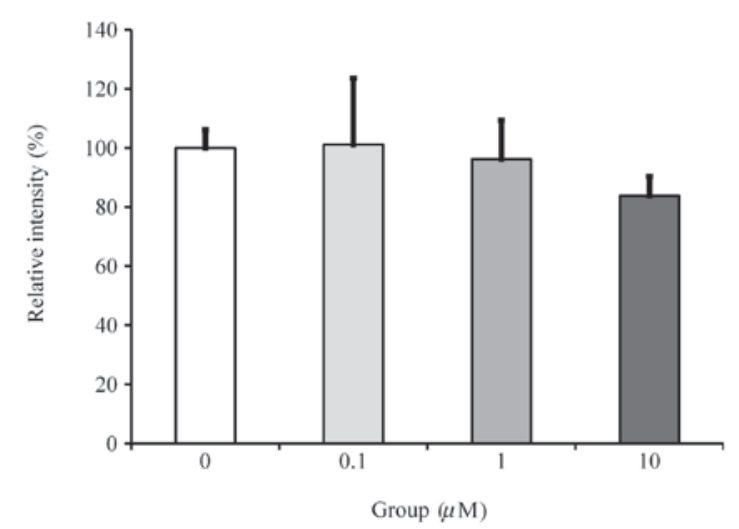

Figure 6. Quantitative analysis of the protein expression of osteocalcin following normalization by densitometry.

$100 \%$, however, this did not reach a statistically significant level $(\mathrm{P}>0.05)$.

\section{Discussion}

The present study examined the effects of low doses of fulvestrant on cell viability, and the differentiation and mineralization of osteoblast progenitor cells under predetermined concentrations $(0.1-10 \mu \mathrm{M})$.

Viability and proliferation were evaluated using the MTT assay and protein concentration. The MTT assay is an extremely sensitive method for determining cell proliferation since this assay allows mitochondrial dehydrogenases to oxidize MTT to an insoluble blue formazan product $(17,18)$. The protein assay may be considered an indirect measurement of cell viability as it measures the protein content of the viable cells remaining after washing the cell plates (19). Equal amounts of DMSO 
were added to each culture sample to offset the influence of the dissolving vehicle (13). The results showed no significant effect of fulvestrant on cell number. Thus, changes in protein expression or mineral levels in the groups may be attributed to changes in the ability of the osteoblasts to produce minerals rather than to changes in the number of cells present $(9,20)$.

ALP activity, which is considered to be an early marker of osteoblastic cell differentiation, was used to determine osteoblast differentiation (14). Cultures grown in the presence of fulvestrant showed a dose-dependent reduction in ALP activity, yet statistically significant differences were not achieved. Osteocalcin is an osteoblast-specific gene expressed by fully differentiated osteoblasts (21). Western blot analysis showed that $10 \mu \mathrm{M}$ fulvestrant yielded a decrease in protein expression, however not at a statistically significant level. In a previous study, osteocalcin expression was significantly reduced in $0.1 \mu \mathrm{M}$ fulvestrant at Day 28 , which may be the result of the longer period of culture (9).

Moreover, cultures grown in the presence of fulvestrant presented with a dose-dependent reduction in mineralization with a statistically significant difference at $10 \mu \mathrm{M}$ concentration. However, previous studies have showed that treatment with fulvestrant alone at either 0.1 or $10 \mu \mathrm{M}$ did not result in significant changes in mineralization (9). The various responses to fulvestrant may be attributed in part to the culturing period, the stage of differentiation of the cells or the culturing condition (22).

The effect of fulvestrant on bone mineralization remains controversial $(5,9)$. Fulvestrant reduced bone volume at the proximal tibial metaphysis by $\sim 30 \%$, associated with an increase in osteoclast surface, while the authors suggested that this was attributable to antagonization of the actions of estrogen (23). Likewise, the increase in calcium content by $17 \beta$-estradiol was completely inhibited by the fulvestrant (24), and co-treatment of estrogen and fulvestrant resulted in a significant reduction of mineralization (9). However, bone mineral density in rats was not altered by administration of fulvestrant at anti-uterotrophic doses, unlike the significant reduction that occurred following an ovariectomy (25), and mineralization by osteoblastic cells was not altered in an estrogen-deficient environment using calvarial osteoblasts (MC3T3 cells). Further elucidation of the mechanisms by which fulvestrant affects bone may have the potential to improve the clinical management of fulvestrant usage.

These findings support the conclusion that the use of fulvestrant may have negative effects on the mineralization of osteoprecursor cells and that the long-term use of fulvestrant may have detrimental effects on osteoblastic activity.

\section{Acknowledgements}

This study was supported by the Bioethics Expert Development Fund of the Committee for Life in the Archdiocese of Seoul.

\section{References}

1. Dechering K, Boersma C and Mosselman S: Estrogen receptors alpha and beta: two receptors of a kind? Curr Med Chem 7: 561-576, 2000.

2. Song C, Wang J, Song Q, et al: Simvastatin induces estrogen receptor-alpha (ER-alpha) in murine bone marrow stromal cells. J Bone Miner Metab 26: 213-217, 2008.
3. Park JB: Effects of low dose of estrone on the proliferation, differentiation and mineralization of osteoprecursor cells. Exp Ther Med 4: 681-684, 2012.

4. Dauvois S, Danielian PS, White R and Parker MG: Antiestrogen ICI 164,384 reduces cellular estrogen receptor content by increasing its turnover. Proc Natl Acad Sci USA 89: 4037-4041, 1992.

5. Curran M and Wiseman L: Fulvestrant. Drugs 61: 807-814, 2001.

6. Osborne CK, Wakeling A and Nicholson RI: Fulvestrant: an oestrogen receptor antagonist with a novel mechanism of action. Br J Cancer 90 (Suppl 1): S2-S6, 2004.

7. Fawell SE, White R, Hoare S, Sydenham M, Page M and Parker MG: Inhibition of estrogen receptor-DNA binding by the 'pure' antiestrogen ICI 164,384 appears to be mediated by impaired receptor dimerization. Proc Natl Acad Sci USA 87: 6883-6887, 1990.

8. Nicholson RI, Gee JM, Manning DL, Wakeling AE, Montano MM and Katzenellenbogen BS: Responses to pure antiestrogens (ICI 164384, ICI 182780) in estrogen-sensitive and -resistant experimental and clinical breast cancer. Ann NY Acad Sci 761: 148-163, 1995.

9. Brennan O, O'Brien FJ and McNamara LM: Estrogen plus estrogen receptor antagonists alter mineral production by osteoblasts in vitro. Horm Metab Res 44: 47-53, 2012.

10. Lykkesfeldt AE, Larsen SS and Briand P: Human breast cancer cell lines resistant to pure anti-estrogens are sensitive to tamoxifen treatment. Int J Cancer 61: 529-534, 1995.

11. de Cupis A, Noonan D, Pirani P, Ferrera A, Clerico L and Favoni RE: Comparison between novel steroid-like and conventional nonsteroidal antioestrogens in inhibiting oestradiol- and IGF-I-induced proliferation of human breast cancer-derived cells. Br J Pharmacol 116: 2391-2400, 1995.

12. Robinson JA, Harris SA, Riggs BL and Spelsberg TC: Estrogen regulation of human osteoblastic cell proliferation and differentiation. Endocrinology 138: 2919-2927, 1997.

13. Park JB, Zhang H, Lin CY, et al: Simvastatin maintains osteoblastic viability while promoting differentiation by partially regulating the expressions of estrogen receptors $\alpha$. J Surg Res 174: 278-283, 2012.

14. Park JB: The effects of dexamethasone, ascorbic acid, and $\beta$-glycerophosphate on osteoblastic differentiation by regulating estrogen receptor and osteopontin expression. J Surg Res 173: 99-104, 2012.

15. Park JB: Combination of simvastatin and bone morphogenetic protein-2 enhances differentiation of osteoblasts by regulating the expression of phospho-Smad1/5/8. Exp Ther Med 4: 303-306, 2012.

16. Park JB: Effects of fibroblast growth factor 2 on osteoblastic proliferation and differentiation by regulating bone morphogenetic protein receptor expression. J Craniofac Surg 22: 1880-1882, 2011

17. Park JB: Effects of doxycycline, minocycline, and tetracycline on cell proliferation, differentiation, and protein expression in osteoprecursor cells. J Craniofac Surg 22: 1839-1842, 2011.

18. Almazin SM, Dziak R, Andreana S and Ciancio SG: The effect of doxycycline hyclate, chlorhexidine gluconate, and minocycline hydrochloride on osteoblastic proliferation and differentiation in vitro. J Periodontol 80: 999-1005, 2009.

19. Fotakis $G$ and Timbrell JA: In vitro cytotoxicity assays: comparison of LDH, neutral red, MTT and protein assay in hepatoma cell lines following exposure to cadmium chloride. Toxicol Lett 160: 171-177, 2006.

20. Qu Q, Perälä-Heape M, Kapanen A, et al: Estrogen enhances differentiation of osteoblasts in mouse bone marrow culture. Bone 22: 201-209, 1998.

21. Qi H, Aguiar DJ, Williams SM, La Pean A, Pan W and Verfaillie CM: Identification of genes responsible for osteoblast differentiation from human mesodermal progenitor cells. Proc Natl Acad Sci USA 100: 3305-3310, 2003.

22. Quarles LD, Yohay DA, Lever LW, Caton R and Wenstrup RJ: Distinct proliferative and differentiated stages of murine MC3T3-E1 cells in culture: an in vitro model of osteoblast development. J Bone Miner Res 7: 683-692, 1992.

23. Gallagher A, Chambers TJ and Tobias JH: The estrogen antagonist ICI 182,780 reduces cancellous bone volume in female rats. Endocrinology 133: 2787-2791, 1993.

24. Sato K, Nohtomi K, Shizume K, et al: 17 beta-estradiol increases calcium content in fetal mouse parietal bones cultured in serum-free medium only at physiological concentrations. Bone 19: 213-221, 1996.

25. Wakeling AE: The future of new pure antiestrogens in clinical breast cancer. Breast Cancer Res Treat 25: 1-9, 1993. 